\title{
Arkadiusz Gut
}

The John Paul II Catholic University of Lublin

\section{Robert Mirski}

The John Paul II Catholic University of Lublin

\section{IN SEARCH OF A THEORY: THE INTERPRETATIVE CHALLENGE OF EMPIRICAL FINDINGS ON CULTURAL VARIANCE IN MINDREADING ${ }^{1}$}

\begin{abstract}
In this paper, we present a battery of empirical findings on the relationship between cultural context and theory of mind that show great variance in the onset and character of mindreading in different cultures; discuss problems that those findings cause for the largely-nativistic outlook on mindreading dominating in the literature; and point to an alternative framework that appears to better accommodate the evident cross-cultural variance in mindreading. We first outline the theoretical frameworks that dominate in mindreading research, then present the relevant empirical findings, and finally we come back to the theoretical approaches in a discussion of their explanatory potential in the face of the data presented. The theoretical frameworks discussed are the two-systems approach; performance-based approach also known as modularity-nativist approach; and the social-communicative theory also known as the systems, relational-systems, dynamic systems and developmental systems theory. The former two, which both fall within the wider modular-computational paradigm, run into a challenge with the cross-cultural data presented, and the latter - the systemic framework - seems to offer an explanatorily potent alternative. The empirical data cited in this paper comes from research on cross-cultural differences in folk psychology and theory-of-mind development; the influence of parenting practices on the development of theory of mind; the development and character of theory of mind in deaf populations; and neuroimaging research of cultural differences in mindreading.
\end{abstract}

Keywords: cross-cultural, folk psychology, two-systems, modular-nativist, developmental systems, social-communicative, mindreading, theory of mind, child development.

The recent increase in empirical findings on cultural variance in theory of mind has produced a diverse body of fragmentary explanations from a number of theoretical positions. Accommodating the growing number 
of cross-cultural findings poses an interpretative challenge for researchers studying the development and functioning of social knowledge based on theory of mind (Kallberg-Schroff M. \& Miller J.M., 2014; Wellman, H. M., Fang, F., \& Peterson, C. C., 2011). Taking up the challenge entails, on the one hand - a comprehensive tabulation of the available empirical data, including the recent results obtained from diverse cultural environments; and on the other - a search for a theory that would provide an adequate model of the development of social skills that hinge on the ability to understand and attribute mental states to others.

In this article we will therefore present recent empirical findings on the relationship between cultural context and theory of mind, and survey the available theoretical positions, suggesting one that seems to account for the empirical data best. In our search for an adequate theoretical framework, we assume hypothetically that the social-communicative approach can be viewed as a serious candidate with theoretical resources capable of accounting for the empirical data within the area of our interest.

The social-communicative view, which has been proposed inter alia by J. Carpendale, K. Nelson and Ch. Lewis, is historically rooted in the ideas of L. Vygotsky and J. Bruner, while philosophically in those of L. Wittgenstein. The wider theoretical framework of the approach is systems theory (Nelson, 2007; Smith, Weintraub, Oyama, \& Lewontin, 2000; Thelen \& Smith, 2002). ${ }^{2}$ It is an alternative to the strictly cognitive perspective; the view that uses the metaphor of the child as a little scientist and is organised within the modular-computational framework, assuming the existence of core concepts or capacities that are constant over development and immune to cultural factors.

The view in question is often referred to as social because it views mindreading as part of social understanding or, in a different terminology, as part of pragmatic psychology (Nelson, 2006, pp. 77-78, Carpandele and Lewis, 2006). ${ }^{3}$ Proponents of the approach claim that it offers a coherent account of ToM-related abilities, including: a) Cross-cultural differences in ToM; b) the influence of social context (e.g. the number of siblings); c) the influence of forms of communication used by the child and her caretakers; and d) neuronal changes caused by differences in environment. In contrast to purely nativistic views, the systems approach does not conceive of ToM in terms of generating new competences by the cognitive system independently of environmental factors. In fact, the systemic framework applied by the view completely reconceptualizes the issue of nativism and universalism of ToM, which will become apparent in our exposition. 
In the following, we will first present the social-communicative stance and sketch its alternatives, and then present a battery of recent crosscultural empirical data that proponents of the multi-systems claim their view accommodates best. This will set the scene for a compartmentalization of the theories on offer in the final section.

\section{The Systemic Framework}

At its broadest, the social-communicative approach applies the more general dynamical systems theory to development. The approach stresses the embodiment and situatedness of the subject; cognition is not seen as a subject-world relation but rather as a phenomenon emergent in a multirelational system where the organism and aspects of the world, as well as cognition itself, are elements/subsystems. Changes in the system and its components that lead to the development and functioning of ToM (and any other cognitive ability) proceed via consecutive bidirectional causalityone element changes another, whose change in feedback changes the original one, as well as possibly some others. This way the system in which cognition develops readapts itself in its entirety in response to change in any of its parts. It is impossible in this view to separate any element or any relation of two elements from the dependencies of the whole structure. Neither is there a pre-set developmental plateau towards which the system progresses, but rather constant readaptation of the system in response to changes in its parts. Moreover, the changes are marked by gradualism - any clear-cut stages of development claimed are merely nominal, while de re they are vigilantly seen as continuous (Carpendale \& Lewis, 2006).

Nelson proposes that cognition and development can be construed as happening within the structure of six subsystems or "constraints" ecological, social, cultural, evolved and embodied, and the one of past experience (Nelson, 2007), which will be discussed in more detail later. As has been said, each of the elements impacts the others; they do not exist in separation but as a function of their mutual relation; they do not have any truly inherent properties. Research within the framework boils down thus to tracking the dynamic change of the system that leads to the emergence of particular cognitive capacity - in our case: Theory of mind. 


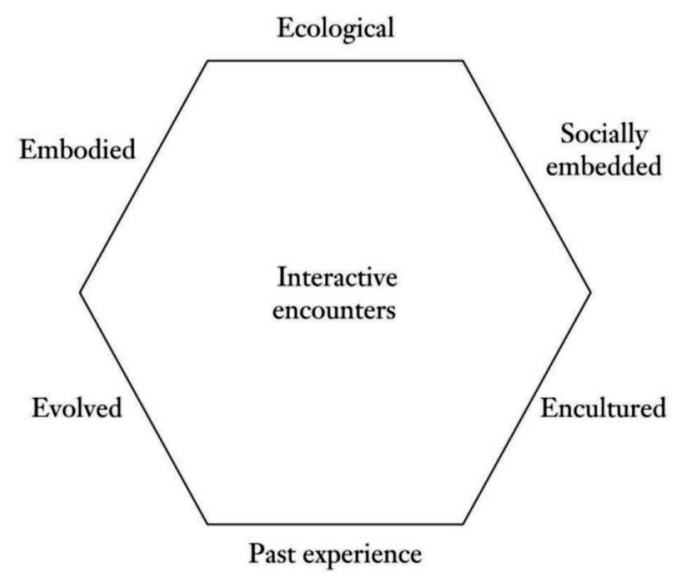

Figure 1. Constraints on experience and meaning within individual interactive encounters (Nelson 2007, p. 19)

\subsection{What is at the forefront?}

The issue of language and culture's place in the account of ToM brought out by the recent proliferation of cross-cultural studies creates an interpretative challenge that has been eluding any firm theoretical grasp. Given the problems that other approaches encounter in the face of the cross-cultural findings, we would like to offer a preliminary exploration of the possibilities that the systemic theory affords. While cultural factors have been much neglected by the computational spirit in which ToM studies have been usually done so far, they are central to the social (pragmatic) take on development.

Studies on mindreading have identified a set of its contributing factors: Inborn concepts, cognitive heuristics, memory resources, executive function, language, and environment (caretakers, siblings, parenting practices, etc.). What distinguishes particular theories is the question of which one is at the forefront, or, in other words - which one is the major contributor to ToM. The social-communicative approach advances that interaction with other subjects is central; it permeates every part of the system in which human cognition develops, and thus any other kind of activity is necessarily stimulated by and mediated through social dynamics. It is the gateway to language as well as to ToM (Carpendale \& Lewis, 2006).

In other words, ToM development is significantly implicated in factors pertaining to social environment - e.g. attachments, the number of siblings, parenting, styles, or parent-child communication. As becomes apparent, the mind in general is understood here as intrinsically located within the widely understood environment, which is essentially social for humans (Bog- 
dan, 1997; Carpendale \& Lewis, 2006; Nelson, 2007; Białecka-Pikul 2012, Hutto et all. 2011). Citing Chapman's idea of an epistemic triangle, "understanding the development of "cognitive" skills is inseparable from an understanding of how such skills are constructed in social interaction" (p. 237). Consequently, it is claimed that

within everyday interactions children constantly negotiate with others who may have different beliefs or perspectives. Such negotiations might involve issues like the location or identity of objects, but may also involve social or moral issues, like whether the child should get another cookie for good behavior. Children construct an understanding of how they and other people acquire knowledge of the world and they may achieve comparable levels of development at similar ages because of commonalities in their experience, not because a module is switched on or because the child suddenly realizes that the mind is a representational device (Carpendale \& Lewis, 2006, p 237).

Interaction is the key to ToM development as it gives birth to theories of the mind and heuristics that explain the social world. The model departs from Gopnik, Meltzoff, and Kuhl's (1999) metaphor of the child scientist and commits itself to the one associated with Vygotsky and Bruner-the culturalhistorical child. As a consequence, a sceptical attitude has been adopted towards the nativistic claim that the cognitive development of mindreading abilities is independent of external factors and proceeds according to a strict schedule, in which potential dysfunctions are caused solely by brain damage or disorders.

Accordingly, the role of language in relation to mindreading is viewed here in quite a different light than in other theories; instead of the universalistic focus on particular, separate aspects of language, like sentential complements or understanding mental concepts, that characterize classical accounts, the emphasis falls on praxis of language and the form of communication that the child takes part in. As Donald (2006) points out, language "emerges only at the group level and is a cultural product distributed across minds" (p. 11). As the child develops, the most significant aspect of the system already discussed - social interaction-becomes increasingly linguistic, and therefore language too comes to be intimately implicated in virtually all other parts of the system, including ToM.

\subsection{Community of Minds as the key metaphor of the theory}

In Nelson's view, cognitive development is "an expansion of the potential for meaningful experience of different kinds, or on different levels" (Nelson, 2007, p. 244). Memory plays a central part in this process, as it is in the light of what has been experienced before that the current experience 
takes on meaning. This, in turn, joins memory too and potentially changes the meaning of experiences to come. And so on. Meaning is defined here as the relevance of experience in relation to any of the contextual constraints (Nelson, 2007). Accordingly, many experiences are meaningful owing to the evolved constraints on experience - e.g. food draws attention - whereas others become meaningful along scaffolded cognitive development- - e.g. words of one's language as a consequence of their growing salience with every single use experienced. Therefore, both cultural and biological constraints on memorized experience together - drawing a clear dichotomy of the two belies the reality of their interaction - play their joint part in meaning formation. As Nelson has it, "experience acts as an interface between the internal and the external components of an integrated system, where embodiment and inheritance play a major role in what can be experienced in a particular setting, and meaning in memory largely influences what will be experienced within that constraint" (Nelson, 2007, p. 249).

Nelson argues that it is best to view the growth as expansion of consciousness because the drastic changes of meaningfulness of experience are said to change what is lived through completely. She draws up six developmental stages that mark significant changes in consciousness. Their brief presentation in the following paragraphs will make explicit the growing centrality of language and culture to human cognition. As can be observed, the majorly innate proclivity for social interaction grows more and more linguistic with every experience that joins memory.

(i) Fresh after birth, the child is said to possess basic awareness, the meaning of which is constrained by innate factors - "the predispositions of the neural structures that, for example, expect and look for 'mother,' for shapes and objects, for language patterns, and so on" (Nelson, 2007, p. 245).

(ii) After a time, these take the child to the level of social consciousness, which is the result of the child's innate attention to its caretakers. People become distinct parts of experience and the child's focus zeroes in on their behavior. Nuances of others' actions are being discerned, which brings out attention to language use and sets the child on the path of language acquisition. This preliminary social understanding enables the child to begin to recognize herself as a person.

(iii) Cognitive consciousness is the third stage singled out by Nelson. The child now functions as an active subject who is aware of the effect her actions have on others and on her own experience. She shares meanings through language and uses it to talk about her own actions. She externalizes meanings and establishes further connections with meanings of others. Language is brought out as the scene where others are met. 
(iv) The appreciation of this intersubjective significance of language marks another drastic change in consciousness. Interaction in language becomes central in experience and the child starts to reflect internally on her, now significantly linguistic, meaning of experience, extending the capacity for abstract thought.

(v) As this development is elaborated, the child masters narrative thought - it becomes possible to think and talk about past, future, and hypothetical situations. Stories of other people, even strangers or imaginary ones, can be now told and comprehended. The distinction of episodic memory and semantic memory is drawn as the child is forced to make sense of the complex net of meanings that assail her from every direction. As a consequence, a mature self is established, unique in its inherently "public" identity. And the access to cultural narratives - which are full of other minds, of their beliefs, feelings, and thoughts and differing perspectivesamplifies ToM development.

(vi) The world is now permeated by shared meanings of culture; the child enters the shared space of what Nelson dubs "the community of minds."

As we can see, there are various types of consciousness in this view; cultural consciousness developed in the last stage is built sequentially first on basic awareness, then on social consciousness, and then on cognitive and reflective consciousness. And finally, narrative consciousness lays the crucial basis for mature mindreading. In short, a full entrance into the world of other minds is necessarily cultural - it demands mastery of understanding narratives and the social world. As Nelson has it,

the most important distinction between the community of minds view and other current views of ToM is that the burden of constructing the model of minds does not rest on the child's individual cognitive powers; rather, it is a gift from the larger community, which incorporates the constructs into its language and its talk about the concerns of people within the community. The child must do work to unpack the gift, which consists of many layers of concepts wrapped within one another, but the rewards are there for the taking if the child is able to try out the ideas for herself. Thus the process of entering a community of minds is necessarily a collaborative one, enabling the child to enter fully into human cultural life. Its beginnings in the early years prepares the child for participation in as yet unanticipated cultural communities in the future (2007, pp. 221).

In keeping with what has been said before, Nelson's sequence shows that from the second stage onwards, language and symbolic interaction play an increasingly more profound role in the child's cognitive development. 
The approach assumes that only within the final environment constituted by the representational use of language can conscious reflection be freed from the limits of actuality - using language in this way makes it possible to think and talk not only about what there is, but also about what was or that which could or will be, as well as to share meanings with other minds and consider their perspectives. What has been studied under the label of theory of mind is in fact the essential quality that underwrites the whole of experiential environment in that final stage. It is part and parcel of the context that meanings are public; that is, available in many ways to other minds. This cognitive calibration that synchronizes one's meanings with those of the community is viewed as a consciousness of a completely different nature than that of the private mind with incomplete cultural binding. Theory of mind, abstract concepts, the cultural self and possibly many other phenomena are seen as emergent only in a system of cognition embedded in culture. The mental does not exist outside cultural or at least social experience; the child's contact with other minds happens only in the sphere of shared meanings.

In sum, the central tenet of the presented view is that cultural experience is indispensable for experience to carry meanings pertaining to the mental. In the next section we will have a closer look at how exactly language and culture are seen in the theory.

\subsection{Pragmatic perspective on language and culture}

As we have said, language grows to be the main tool for social interaction that permeates the system of human experience, and hence the proponents of the pragmatic view see it as intimately implicated in the development of many cognitive capacities as social interaction itself is. In other words, the assumption is that language mediates children's understanding of the mind by providing criteria for identification of the mental world (Carpendale \& Lewis, 2006).

Specifically, the social-communicative approach is characterized by a Wittgensteinian stance on language, which is a natural move considering the theoretical framework adopted-Wittgenstein's idea of language chimes best with the systemic theory. From this pragmatic perspective, language is viewed as a communal activity: Its meaning seen as the outcome of experience with discourse; and learning words and expressions as about homing in on their criteria of use practiced in the society, not about mapping them onto their referents (Carpendale \& Lewis, 2006; Montgomery, 1997; Nelson, 2007). 
The upshot of such understanding is that linguistic meaning, similarly to any other meaning in the described developmental system, comes about as a consequence of experience with the use of linguistic forms; meanings understood and intended are derived as the function of experience with language constrained by the six factors proposed by Nelson. For example, the child will more likely pay attention to words that are already present in her memory (past experience), or that are highlighted by the parent (social embedding). Every new experience adds to memory and thus changes the meaning of another experience. In this way, language is learnt in the pragmatic conception.

\subsection{Concepts and language}

Related to language, and central to ToM research, is the idea of concepts. The core assumption here is that their acquisition boils down to recognition of patterns in experiences and formation of categories based on common features of those experiences. As a consequence, it seems possible with this view to acquire concepts that refer to the observable reality without the involvement of language. What is required is just enough experiential data to draw on and extract the array of related features, and "bind" them to the most salient one that comes to represent them in thought. Naturally, words and expressions used will be the most salient common denominator of much of perceptual experience and thus, together with the other perceptual elements accompanying them (which make for their criteria for use), will facilitate the formation of many concepts. Take temporal sequentiality, for example; it seems entirely possible that the corresponding concept be formed solely as the result of the repeated experience of the relation of one thing happening after another. However, language is said to help greatly as "the use of the words to express specific instances of these relations may aid in making the relations salient and thus precipitate the child's conceptual understanding" (Nelson, 2007, p. 154).

What is more, externalization of forms of language allows for collaborative "tweaking" of concepts, their adequate reconfiguration with the help of interlocutors (Nelson, 2007, p. 234, 2007, p. 232, 2007, p. 225). Children are reported to first use words in a nonsensical manner, which is interpreted by Nelson as a cognitive strategy to put "the not-yet-comprehended form out into the social space where it can be reflected on and responded to by both others and the self" (Nelson, 2007, pp. 168-169). Such use without meaning provides additional experiential data and, together with the other elements of the developmental system, works to imbue the used words with 
meaning. This includes non-verbal meaningful parts of experience, as well as verbal ones - as the child develops linguistically, acquisition of new words is also facilitated by the context provided by the discourse that is already understood.

The syntax of language contributes too (Nelson, 2007, p. 159). In addition to the contexts highlighted so far, words are uttered in the context of grammatical function. Nelson recognizes this and discusses the influence of syntactic bootstrapping of "slot-filler" categories. In the general conception, syntactic bootstrapping relies on syntactic structure as a clue to the word's meaning (Ruffman, Slade, Rowlandson, Rumsey, \& Garnham, 2003, p. 154). Nelson argues that this process plays a significant role in the formation of superordinate concepts. She draws on the semiotic theory that the syntagmatic spread of language creates "slots" to be filled with certain words and the words that can fit into a particular slot form paradigmatic categories. With experience of such syntactic constructs, superordinate terms come to represent the slot-filler categories, exerting a concept-forming influence on the child's mind. As empirical data shows, young children tend to view superordinate terms as denoting collections of items (since they are usually used this way by parents), not as representing abstract categories of inclusion (Nelson, 2007, p. 159). It takes time to understand that such terms are in the relation of inclusion to other terms, which is argued to come about greatly with the help of syntax. This example is far from exhaustive; it is only aimed to illustrate the point.

Putting it all together, it can be said that the degree of a concept's dependence on linguistic experience can be viewed as resting on how much of the experiential data necessary for its formation is supplied by language. The more abstract the concept, the greater its reliance on conversational experience. Consequently, in the case of many abstract notions, such as the mind, there is no other experience apart from the conversational one on which to draw, and hence language and social interaction are seen as indispensable for their acquisition - they exist only within socio-linguistic contexts (Pyers \& Senghas, 2009; Wellman, H. M., \& Peterson, C. C., 2013). As Nelson contends, it is "through language that the child has access to conceptual systems that are embedded in and thus only accessible through linguistic forms, not through direct experience" (Nelson, 2007, p. 151). However, by no means should this formulation downplay the role of other factors; the dynamic formational process involves language in its many aspects as well as social interaction per se and maturing cognitive capacities too-they are all engaged in a multicausal interplay which results in the formation of what falls under the label of theory of mind. 


\subsection{Other takes on language}

As we have seen, the pragmatic approach takes language together with other substructures present in the system and views them as inter-defined. From this holistic perspective, it becomes possible to trace how particular changes impact the whole system and its emergent elements, such as ToM. The view needs to be contrasted with other approaches that can be described as more essentialist or atomistic and study the language-ToM relationship in separation, seeking what there is in language understood as a kind that relates to ToM. Theories in that spirit tend to inquire into language as an internalized system, a cognitive tool that by virtue of its structure facilitates ToM development.

A good example of such an approach is that of studies conducted by de Villiers et al. who have argued that it is the combinatorial properties of language that enable the child to reason in new ways about others' beliefs. Specifically, mastering the grammar of complementation is said to provide a cognitive tool, a new format, for a proper representation of (false) beliefs (Villiers, 1995; Villiers, 2000; Villiers \& Villiers, 2000; Villiers, 2005; Villiers \& Pyers, 2002; Villiers \& Villiers, 2014; Villiers \& Villiers, 2009). This model points to syntax as much as it does to semantics - it is not the syntax of complementation per se that exerts its influence on the child's mind, but its combination with the semantics of the embedded clauses. The value lies in contact with overtly false embedded clauses through which the child learns that the subject can say something false, and also think it (e.g. Mommy says/thinks that it's raining outside [in fact, it's sunny]). The structure together with the specific content does the trick. Consequently, not every kind of complementation is said to be significant (e.g. irrealis complements like the ones taking the verb want or promise) (Milligan, Astington, \& La Dack, 2007, p. 640; Villiers \& Pyers, 2002). There is much research that supports this theory (Hale \& Tager-Flusberg, 2003; Tager-Flusberg \& Joseph, 2005; Villiers \& Pyers, 2002; Gut A., 2009).

The problem with the above line of argumentation, however, is that when one posits that it is language as an internalized cognitive tool that matters, one is obliged to demonstrate the way in which it does so - a lost cause in the face of data showing that virtually every aspect of language is related to ToM-tasks performance to some extent. The systemic approach, on the other hand, avoids that charge by stressing the embodiment of cognition and construing concepts as inseparable elements in a complex system (Nelson, 2007, pp. 38-39; Tafreshi \& Racine, 2016, p. 58). Cognitive development within that framework is seen as a composite multicausal process where it is impossible to establish simple cause-effect relations between el- 
ements. ToM is thus seen as a phenomenon emergent in the environment consisting of social life and other elements of the cognitive system; it is entangled in a multidirectional dynamic, being caused by many factors and in feedback changing them as well, and potentially leading to the emergence of others. Hence, what has been described as the precursor developments and significant aspects of language are still naturally needed for the formation of ToM, but they do not lead to nor constitute any proper conceptual theory of mind by themselves - even an implicit one (Nelson, 2007, pp. 214215). Arguably, the full picture of ToM development can be only drawn with the consideration of the whole of the developmental environment; and that is why the role of language is stressed - it is the fabric of the human milieu.

Locating language in social practice and assuming that language is part of the activity that supplies criteria for identification of the mental world means a departure from universalistic views on language that characterize approaches done within the modular-computational theory of cognition. As has been already stated, such views concentrate on the features of language that are shared by all languages, that are necessary conditions of something classified as a language. There are two ways of tackling the differences in ToM in this view; either one claims that the structure of language as an internalized cognitive tool differs across cultures and impacts ToM understanding conceptually, or that variance in the structure affects only explicit voicing of ToM understanding that is essentially already there by nature.

\subsection{Two views in the modular-computational spirit}

The universalistic views usually postulate that knowledge of the developing child is organized in a theory-like way. Along with the child's theories about the world's physics, biology, or linguistics, the child is also assumed to form a psychological theory. Usually, such theories posit the existence of an inborn concept of the mind, or some basic theoretical structure pertaining to the mental that comes in the package of a brand new brain.

From the nativistic view, language's significance is much weaker as its virtue lies in either merely enabling the child to apply the inborn theory to language-embedded situations, or providing a new format for computing psychological data, extending the theory significantly with conceptual overgrowth instigated by language. The latter option, in other words, assumes the preexistence of some inborn knowledge or module dedicated to processing psychological information, which is then greatly developed by acquiring language (Callaghan et al., 2005, p. 383). Usually, a significant separation 
is assumed between the two kinds of psychological knowledge -innate and cultural-which has come to be referred to as the two-systems approach. Proponents of the fully-inborn view, on the other hand, posit children's early full understanding of false beliefs, and blame the failure in the verbal false-belief tasks on their linguistic and computational demands that do not match the domain-general capacities of children before 3-5 years of age (Milligan et al., 2007, p. 637). Children implicitly understand other minds, but pragmatic issues with language stand in their way of voicing it. This view can be labeled as performance-based or one-system.

The two alternatives seem problematic and the inclusive picture of language in the systems theory seems worth exploration. Before we go into further comparison of the theories, keeping the theoretical assumptions of the pragmatic approach in mind, let us first explore a body of empirical data and consider if there is really something to gain in the new perspective.

\section{Data from Differing Linguistic Environments}

The systemic theory as presented so far, viewing mental concepts as extracted from social interaction, which sometimes is termed mindsharing, falls within the general pragmatic description of how abstract concepts are acquired. The process of acquisition of abstract meanings, and, by the same token, the formation of corresponding abstract concepts, is said to be the outcome of complex socio-linguistic experience - conversations, various social interactions, and contact with other cultural elements. As such, Nelson's theory accounts for research that focused on the function of conversation in ToM development (Harris, 2005; Rosnay \& Hughes, 2006; Ruffman et al., 2003, p. 152; Watson, Nixon, Wilson, \& Capage, 1999, pp. 386-387), extending the perspective to the whole of cultural experience (Nelson, 2007, p. 237), which also colligates studies tackling specifically the relationship between social interaction and ToM performance (Watson et al., 1999). As has been demonstrated, research centering on syntax is also included. Naturally, the development of executive function and other maturational cognitive capacities also fits the picture, as does the possibly inborn mindreading module (which is, however, not seen as providing the concept of mind by itself).

The available data will be presented in the following order. Points 2.1 to 2.3 will present data from three studies demonstrating significant crosscultural differences in the development and functioning of ToM. Next, we will review a battery of neuronal research and we will address findings deal- 
ing with parent-talk influence and also ones conducted on deaf subjects. We intend to present the data with special regard to details significant for empirical research. In recent decades, there have been a growing number of studies focused on differences in ToM development across cultures, and yet the data is still far from being wide-spread.

\subsection{The Indian case}

In their 1994 study, Wahi and Johri tested young Indian children's ability to make real-mental distinctions. Their aim was to (a) try to replicate previous studies that had shown consistent aptitude for such distinctions in young children, regardless of the object's kind, and (b) test the potential influence of Indian culture and difference from data collected in the USA.

First of all, the kind of object did affect the children's ability to distinguish it from its mental representation. From the three categories testedtoys, concrete objects, and celestial objects - toys turned out to be the easiest, and celestial objects the hardest. This finding disagrees with previous research demonstrating the irrelevance of kinds of objects in making mentalreal distinctions by young children. On the other hand, it does agree with the Piagetean theory in that children found it the easiest to reason about concrete objects, but at the same time clashes with it in that the domain (toys vs. regular concrete objects) did matter.

The authors provide a few explanations. Toys are argued to be easier in virtue of the experience that the child has had with them playing (Wahi \& Johri, 1994, p. 508). For example, the pretend play exercised with the use of a doll may give a child more practice in making real-mental discriminations about the specific object as well as the whole domain of "toys."

On the other end of the scale, the reason given for the difficulty with celestial objects distinction is twofold. First, the remoteness and perceptual inaccessibility of stars, clouds, or the moon may confound children's conceptualization of them as something real. Second, the influence of culture that often shrouds celestial objects in myth and magic is seen as a possible cause of children's problems with establishing their status.

The second significant finding was that the majority of Indian children successfully made the distinctions only after age 5, contrasting with 3-yearolds passing in the studies from the USA. This is again argued to be the result of Indian culture that can possibly put less emphasis on metacognition about physical objects than the West. It is possible that children in India would be better at metacognizing about the interpersonal interaction that their collectivist culture emphasizes. This remains to be seen as the study did not include such categories. Nevertheless, the fact remains that the study 
provides ground to think of real-mental distinction ability as attained in, or at least affected by, cultural experience.

\subsection{The Samoa case}

Callaghan et al. conducted a study aiming at gathering commensurable data from 5 cultural environments (Canada, Peru, India, Thailand, and Samoa) (Callaghan et al. 2005). What they found was an apparent synchrony in the onset of mental-state reasoning, which was interpreted as supporting universalism. This, however, has been questioned by another study.

The seemingly universalistic results of Callaghan et al.'s 2005 work served as an inspiration for another study that addressed a weak link in the universalistic conclusion that the authors made. The purported synchrony was the weakest in respect to the children from Samoa, with only 18 out of 25 passing at age 4, and 13 out of 18 at age 5, which led Mayer and Trauble to conduct another study dealing specifically with Samoan children to get a closer look at the nature of the difference (Mayer \& Trauble, 2012, p. 22). Before presenting the results and ensuing discussion, let us first outline the cultural context of the Samoan culture that is significant for this paper.

Samoa is one of the Pacific cultures characterized by ideology termed by Joel Robbins and Alan Rumsey as a doctrine of the opacity of other minds (Joel Robbins \& Alan Rumsey, 2008). The Samoan culture is permeated by the view that it is almost impossible to know what is on another person's mind. Samoans are reluctant to speak about others' minds as well as their own, refusing, for example, to give reasons for their own actions (Alessandro Duranti, 2008; Mayer \& Trauble, 2012, pp. 22-23). In a stark contrast to the Western world, social interactions in the Pacific (incl. Samoa) are carried out under the assumption of the impossibility of knowing mental contents, as the opacity doctrine is "a widely shared and taken-for-granted fact about the world, and one that shapes normative orders and everyday practice" (Joel Robbins \& Alan Rumsey, 2008, p. 411).

Another fact that is precious in its own right is that there is extensive social interaction among Samoan people determined by the social environment:

Instead of closed houses, open fale, which have no walls but a roof on wooden columns, are traditionally the centre of life where people meet, cook, eat and work. The open structure of Samoan villages enhances social interaction. Family households are spatially organized together in a single compound. Children in Samoa grow up in the middle of their 'aiga; that is, the extended family including uncles, aunts, cousins, and so forth. Samoan children can use the 
word tin $\bar{a}$ (mother) for all female relatives in the mother's generation. It is quite common that some children do not sleep in the same house with their biological parents, but in the house of someone else who belongs to the 'aiga. All these factors create a populated environment and Samoan children learn to interact with several members of the extended family early on in life (Mayer \& Trauble, 2012, pp. 22-23; Ochs, 1988).

This makes the Samoan an interesting subject of study as on the one hand, the amount of social interaction should promote better false-belief understanding, while on the other, the anti-mentalistic ideology should thwart it. Therefore, the results of the false belief tests serve as evidence for which of the factors contributes more to the formation of ToM.

The results of Mayer and Trouble's 2012 study were that it was not until 8 years old that a majority of the children tested passed the false belief task, and some of them did not do so as late as 12 years (Mayer \& Trauble, 2012, p. 26). This finding runs against Callaghan et al.'s 2005 claim that the previous cross-cultural asynchrony of onset of mental-state reasoning was an artifact of varying experiment methods, and once again casts doubt on the purported universality of mental-state reasoning development (See Callaghan et al.'s 2005). Additionally, the rich social experience that Samoan children get seems to be a less significant ToM contributor on its own without the accompaniment of the right language.

\subsection{ToM scale studies}

Another batch of cross-cultural studies that needs to be considered are the ones utilizing the ToM scale methodology. ToM scale is a measure of a number of ToM developments proposed by Wellman and Liu (2004) to overcome the limitations of studies measuring only a single ToM expression - false-belief understanding. The tool assesses the understanding of "(a) diverse desires (people can have different desires for the same thing), (b) diverse beliefs (people can have different beliefs about the same situation), (c) knowledge-ignorance (something can be true, but someone might not know that), (d) false belief (something can be true, but someone might believe something different), and (e) hidden emotion (someone can feel one way but display a different emotion)" (Wellman, Fang, Liu, Zhu, \& Liu, 2006). The tasks are standardized to be similar in form, and all hinge on the general concept that subjective mental states can contrast with objective events or behaviors. Therefore, considering how the tasks do differ, the sequence that the child progresses on the scale can tell a lot about the nature of ToM development. Clearly, comparison of ToM scale results from different cultural environments carries a promise of great insights. The scale 
has the methodological advantage that it measures the sequence of development, which renders it independent from age or other absolute measures that are likely to confound cross-cultural studies. In this section we will present results from ToM scale experiments coming from different cultures.

First of all, we present facts from studies conducted on children from the West. The sequence confirmed for English-speaking children is as follows: Diverse desire (DD) $>$ diverse beliefs $(\mathrm{DB})>$ knowledge-ignorance $(\mathrm{KA}-$ knowledge access $)>$ false belief $(\mathrm{FB})>$ hidden emotion $(\mathrm{HE})$. The age range of this development is 3 to 11 (Wellman \& Liu, 2004). Importantly, although delayed, the sequence was consistent in the case of deaf children of hearing parents (Wellman, H. M., \& Peterson, C. C., 2013, p. 60, Gut A. \& Wilczewski M., 2015).

In 2006, Wellman et al. conducted a study that applied ToM scale tasks to Chinese children and compared the results to those from the previous study on English-speaking children. The difference they found was the reversed $\mathrm{KA}$ and $\mathrm{DB}$ order: Children from China followed the sequence "DD $>\underline{\mathrm{KA}}>\mathrm{DB}>\mathrm{FB}>\mathrm{HE}$," which contrasts with the American "DD $>\underline{\mathrm{DB}}>\mathrm{KA}>\mathrm{FB}>\mathrm{HE}$ " on the order of these two tasks. The initial crosssectional findings have been supported by a longitudinal study (Wellman, Fang, \& Peterson, 2011). Additionally, another study found the same sequence of progression in children from Iran (Shahaeian, Peterson, Slaughter, \& Wellman, 2011). A number of explanations have been given so far.

The main line of explanations given is that Chinese and Iranian cultures are collectivist, with the cultural emphasis on "knowing, and on children acquiring practical knowledge" (Wellman et al., 2006, p. 1080), as opposed to the Western individualist emphasis on "truth, falsity, and differences in belief" (Wellman et al., 2006, p. 1080). Consequently, children from these cultures have different social, linguistic and parental experiences with their distinctive highlights that are said to impact what kind of conceptual development children follow (Wellman et al., 2011, p. 782). The individualist bias of the West promotes DB understanding before KA - "every person is different and has different beliefs", while the collectivist respect for knowledge and authority is said to do the opposite.

Wellman et al.'s $(2006,2011)$ explanation evokes two systems. On the one hand, they propose that there are universal cognitive capacities, inborn or universal experientially across cultures and necessary for further ToM development. Understanding desires may represent such a capacity as for all children tested DD was the first task passed. On the other hand, children are hypothesized to develop different cognitive emphases built on the universal ToM capacities that follow from the culture's conceptual focus 
on different applications of the common subjective-objective intuition. The knowledge-focused experience particular to China will promote recognition of knowledge-ignorance distinction first, whereas western individualism will promote diversity of beliefs among people.

\subsection{Neuronal argumentation}

Further important evidence, however limited, comes from neuroimaging research. Kobayashi et al. (2006) conducted a study measuring cross-cultural differences in the neural correlates of ToM. Previous neuroimaging research studying the relation between ToM correlates and language correlates has produced mixed results. On the one hand, patients with aphasia have been demonstrated to retain mindreading while having severe linguistic disorders. On the other, activations in areas normally dedicated to language have been shown to be engaged in some ToM processing (Kobayashi et al., 2006, p. 211). The authors conclude that the involvement of language (and culture) in ToM varies in its character and scope depending on the particular aspect of language considered; they suggest that some aspects (e.g. grammar) may be only co-opted, while others (e.g. pragmatics) may significantly impact ToM.

Although there has been plenty of research examining the neural correlates of ToM, only two studies were carried out on non-English speaking subjects, and the two did not make any cross-cultural comparisons (Kobayashi et al., 2006, p. 211). Kobayashi et al. (2006) addressed this gap in evidence and conducted a study aimed at measuring neural differences in ToM processing in American English monolinguals and Japanese-English bilinguals. The study group included 16 adult monolingual Americans speaking English, and 16 adult individuals who spoke Japanese as their first language and English as their late second language. Functional magnetic resonance imaging (fMRI) was used and neural activity was measured in English and Japanese second-order false-belief ToM stories, non-ToM control stories, and scrambled sentences (Kobayashi et al., 2006, p. 211). The study sought to explore possible cultural and linguistic variations in the neural correlates of adults' ToM (Kobayashi et al., 2006, p. 211).

The ToM-related brain areas highlighted by this study are medial prefrontal cortex (mPFC), anterior cingulate cortex (ACC), temporo-parietal junction (TPJ), dorsolateral prefrontal cortex (DFLC), middle frontal gyrus (MFG), post-central gyrus, inferior frontal gyrus (IFG), orbito-frontal gyrus (OFG), and caudate nucleus. The results obtained suggest that some areas employed in ToM processing are shared cross-culturally, while others are specific to a given culture. Besides areas showing clear cross-cultural 
variance, there were subtle differences even in regions that were universally engaged.

As the limited length of this article does not allow us to go over the findings in detail, let us direct anyone interested to the original article and highlight here only the general conclusion significant for our purpose: Some brain regions employed in ToM processing may be evolved, innate, and universal contributors; some may depend on the way other minds are featured in a particular culture; while yet others may depend on the way the internalized language system is co-opted in the processing of the beliefs of others.

\subsection{Influence of parents' talk}

Another valuable piece of evidence for ToM-language discussion comes from studies of parental speech. Since parents are the main source of conversational experience for a young child, examining parent-child conversations is highly informative. There have been a number of such studies that attempted to measure the character of the interaction from various angles. Some of the studies aimed at measuring (i) a particular aspect of parents' speech; others, (ii) tried to map the total of parents' mind-mindedness.

Recently, studies have been conducted that aimed at demonstrating the significance of parents' general conversational focus on mind themes as well as their general attitude towards the child as an individual with a mind - which was termed their mind-mindedness (Carpendale \& Lewis, 2006, p. 164; Dore \& Lillard, 2014, p. 3; Meins, E., \& Fernyhough, C., 2010). The influence of mothers' personal epistemologies on both mental talk measured in the experiments and children's ToM tasks performance has also been examined recently (Tafreshi \& Racine, 2016). We will focus on that research as it subsumes the previous one.

Taken generally, parents' mental term use predicts FB tasks performance and is assumed as a contributor to ToM development. However, as has been discussed earlier, the pure-semantics approach is not tenable anymore. For that reason, recent studies examined use of mental terms that were understood pragmatically; that is, terms were coded for function rather than semantic meaning. Furrow, Moore, Davidge, and Chiasson (1992) found that although mental utterances occurred more frequently in their conversational function rather than mental reference, the later sense still saw a significant increase between 2 and 3 years of age. Moreover, they found that children's mental term use mirrored that of their mothers from the previous stage of the study. Brown, Donelan-McCall, and Dunn (1996) found that children use more mental utterances with their siblings and friends than with parents, the amount of which predicted 
their ToM, suggesting the importance of wider social context for ToM development. Relatedly, the number of the child's siblings positively correlated with her false-belief understanding (Jenkins \& Astington, 1996; Perner, Ruffman, \& Leekam, 1994). Ruffman, Slade, and Crowe (2002) strengthened the causal interpretation of the relationship by controlling for many variables - children's own use of mental state language, their earlier theoryof-mind understanding, their language ability, their age, mothers' education, and other types of mothers' utterances. Most importantly, although they considered various aspects of language, it turned out that it is general talk about mental states that is the best ToM predictor, not its one particular facet. They also found correlations of other aspects of motherchild interaction and ToM development: General descriptions of the stimuli present in the pictures; fact-based teaching; talk about causes; linking talk, in which mothers linked events in the pictures to their children's own life; and elaborative thematic talk that went beyond the content of the pictures themselves - but they were not significant over and above mental state talk.

Studies on what has come to be called parents' mind-mindedness have extended the pragmatic approach to understanding mental talk. Tafreshi and Racine (2016, p. 67) examined mothers' personal epistemologies' (their idea of the nature of knowledge, later referred to as "PE") relation to children's interpretive theory of mind (understanding that people can interpret things in two different, but equally valid ways - identified as a higher-order cognitive capacity of ToM that children pass at around 6-7). Apart from the central finding that the complexity of mothers' PEs significantly contributed to the variance in children's IToM scores (19\%), they found that "mothers with more complex PEs were more likely to elicit talk about interpretive features of the picture book, but they were not more likely to produce talk about interpretation" (Tafreshi \& Racine, 2016, p. 67). This harks back to the discussed role played by children's externalization of learned linguistic forms, and suggests that providing such opportunities for externalization is central in the purported mind-mindedness significance, rather than mothers' own production of mental talk.

\subsection{Data from studies on deaf children}

Another source of valuable data for cultural dependence of ToM is studies on deaf children. First, the linguistic environment of deaf children is in many ways different from that of hearing children, which provides a useful comparison. And second, they do not have any central neurological deficits that could be blamed for delays in ToM understanding, as is the case with 
children with autism. Hence, any such delays if they occur can be interpreted as reflecting the children's lack of particular social or linguistic experience, specific to their situation. In this section we will present insightful research coming from that area (Callaghan et al., 2005, p. 382).

As has already been noted, the studies with the use of ToM scale demonstrated that deaf children of hearing parents progress according to the sequence specific to their culture as do hearing children, but are significantly delayed and the development is possibly incomplete (Wellman et al., 2011, p. 782). This is not true about deaf children who were born into nativesigning families - they progress at the same pace as hearing children. Wellman, H. M., \& Peterson, C. C. (2013) conclude that the reason for the difference is that despite their efforts, non-native signer parents are not capable of providing mental talk of the complexity required to progress at the standard speed. Since such children do not usually differ in the amount of social interaction but only in the quality of linguistic experience, the evidence suggests that there is something in linguistic interaction per se, not just social interaction, that significantly influences ToM understanding.

Further, Pyers and Senghas (2009) conducted a unique study with adolescent and adult signers of an emerging sign language in Nicaragua. They measured FB understanding on two groups of subjects at two time points separated by 2 years. The first group comprised of people who first naturally developed the new language. The second were younger individuals who joined later, acquired the early form of the language and expanded it. The early form of the language developed by the first group lacked significantly in forms expressing meanings related to the mind, the second group broadened it in this respect. The results speak strongly in favor of great dependence of ToM on linguistic interaction. At time 1, the first group produced significantly fewer mental signs than the second one and was found to fail the FB task well into adulthood, which was interpreted as demonstrating that mental talk experience is a necessary contributor to ToM development. The claim is additionally strengthened by the fact that in-between the measurement times, the first group started to socialize with the second group and acquired much of the augmented version of the language - which positively impacted their performance on the FB task at time 2 dramatically. Even in their late twenties the first group was able to extract a better ToM from the linguistic experience that was offered to them. Therefore, in addition to supporting the linguistic entanglement of ToM, the results are said to speak against any "critical time" for ToM acquisition as well as any maturational theories connecting ToM with age. 


\section{Conclusions and Discussion}

Assuming the data presented is reliable, we have thus laid the ground for discussion on the cultural entanglement of mindreading. Cultural and lin-

guistic context does impact ToM as measured by the empirical research. The next step is naturally to seek a theory that would give a systematic account of cognitive development including these findings. In the first sections of the text, we have presented the social-communicative approach and suggested its potential for such an account. The reason for that has been that it is a relatively new outlook on cognitive development and the reader may not have been familiar with it, as well as the fact that its proponents promote it as the best suited for the sort of account we are looking for here. We intend this text to provide an explicit expression of the theoretical choices that we are dealing with here - to put the available theories on the table and form a succinct point of departure for further theoretical deliberations.

We may distinguish three general approaches to mindreading in relation to cultural context present in the literature. If we were to sketch a culturenature scale, the social-communicative approach represents the cultural end of the spectrum (although it does not really separate culture from nature, culture still is a great focus there), followed by the hybrid two-systems approach somewhere in the middle, and the other end is occupied by the nativistic one-system approach, which can also be called performance-based considering the interpretation of the empirical data from sections from 2.1. to 2.6. that it affords. In a way, the level of cultural dependence claimed by the views is positively correlated with the number of systems, or entities, responsible for ToM development that they posit. The purely nativistic performance-based approach posits the existence of only one, inborn, mindreading system that is necessary and sufficient for ToM; the two-systems approach, apart from the implicit system, posits another, explicit one and thus allows for the possibility of a culture-based mindreading; and finally, the situatedness- and embodiment-highlighting pragmatic approach adopts a systemic framework that posits a complex net of multiple systems in which ToM emerges. Moreover, the social-communicative approach is advanced within a theoretical structure completely different from the standard cognitive one; it is inspired by the developmental systems theory (Nelson, 2007; Smith et al., 2000; Thelen \& Smith, 2002).

\subsection{Social-communicative interpretation}

The hallmark of the theory is the claim that ToM emerges as a function of many factors. The plenitude of contributors involved has been dis- 
cussed in the first sections of the paper. As has been presented, the development of the cognitive system is seen here as embodied and embedded in environment and characterised by gradualism (Carpendale \& Lewis, 2006). This means that changes in the system happen in a more continuous manner than has been assumed in the other theories. As a consequence, the amount of social interactions with others and their character can speed up or slow down the time at which the child passes the standard ToM tests, which seem to agree with the empirical findings. Of the greatest importance here are those that come from natural environments and demonstrate high variability in the time of passing the tasks (Carpendale \& Lewis, 2006). Attention is brought to some inconsistency between laboratory-based assessment procedures - standard false belief tests, and naturalistic observation of young children (Carpendale \& Lewis, 2006). What is emphasised in this view is the fact that cultural factors do not wait in line, but interact with the biological system from the very beginning. Additionally, the development of the system does not only proceed through internalization, but happens also in-action. Interaction with other minds is central to the systemic approach, which is supported by the numerous data on the influence of the parenting style, the number of siblings, or parent-child communications.

It is important to note at this point that results obtained in research on the differences in ToM scale progression between children from the West and those from the East (Japan, China, Iran) correlate with parenting styles present at home. As has been said, many studies support that Chinese children, unlike children brought up in Western culture, experience collectivist and interdependent cultural practices, i.e. those where "many parents teach filial respect, emphasize the acquisition of well-established knowledge, and encourage children's conformity to the cultural models, rules, and traditions conveyed by their elders rather than self-assertive expression of their own independent points of view" (Shahaeian et al., 2011, p. 1240; Naito, 2003; Naito, 2004). Relatedly, this is linked by some scholars to the fact that Chinese parents often discourage their children from expressing their own opinions or do not give them a chance to express them, and spur them to endorse opinions that are commonly shared and accepted. This casts doubt on any claims that try to account for the data presented in section 2 solely in terms of performance.

The systemic framework departs from the research idea that focuses on the single mind of an individual child as a little scientist, and commits itself to the idea of the social mind entangled in many relations (Carpendale $\&$ Lewis, 2006). The mind is embodied in the world and thus: 
culture surrounds the child even before birth in the practices of prenatal care. The social figures in the child's life are essential to wellbeing, even to life itself. They provide the supportive structures that enable the child, in whatever conditions of culture and ecology, to enter into the life of the social world, as well as to acquire specific aspects of cultural knowledge and practice. Among the most important of these, certainly, is basic oral language, acquired through sharing meaning with others in speech. Language carries culture within it. The members of the child's social world - family, friends, teachers, and so on - serve as cultural carriers through their speech, and through their behavior as well, which reflects the habits of the particular culture within which the child is growing up. Child-rearing practices are designed, consciously or not, to impart aspects of the culture (Nelson, 2007, p. 13).

Having outlined the purported potential of the systemic framework, it is now necessary to consider the two alternatives to the social-communicative view. Let us then briefly refer to the two-systems and one-system accounts and tentatively indicate how their explanatory power in respect to the presented data can be questioned.

\subsection{Two-system account}

One of the two dominant views in the literature is the two-systems approach (Apperly \& Butterfill, 2009). The two systems - called respectively low and high, early and later, or implicit and explicit - are dedicated to interpreting information in mental terms (intentions, plans, desires, or thoughts). The later-developing mindreading system is assumed to be highly flexible and effortful and its emergence and development are seen as happening in interaction with other systems - starting with language, through the involvement of executive functions, and ending with such external systems as the environment of early education. The general assumption is such that the early, universally structured system is later replaced by culture-specific concepts and a culture-specific mindreading system. According to the twosystems proposition, it is assumed that children's exposure to their daily sociocultural context and language-specific factors affect their very corpus of knowledge and understating of the mind. Culture- and language-specific factors are implicated, at the very least, in the explicit system of mindreading. The view radically distinguishes implicit and explicit competence in mindreading. What is radical about this division is that it cannot be explained by invoking variations in executive functions only - the two systems differ by their nature.

The approach lies open to criticism in virtue of its assumption that the biological mindreading system is at some point complemented by the 
cultural one which is, however, external to the former, and there is no real trade-off between the two. Two problematic issues follow from this view. When the cultural system enters the mindreading game either there is some kind of bio-cultural hybrid created, or the cultural system takes over the biological one or replaces it. Both of the options clash with the findings presented in section 2 that show the complex scaffolding and progressive interdependence between the mind and the environment. In other words, the data show rather that the natural constitution of the mind is nourished by cultural factors and that cultural factors are internalized by the biological system, which is pointed out in the works of Carpendale and Nelson who follow Vygotsky's theoretical scheme. The cross-cultural and neuronal research presented seems to support such a stance.

\subsection{Performance-based interpretation}

Another of the two classical accounts is the one-system, or performancebased, approach. It can be also called a weak interpretation that goes as follows: Culture-specific factors have an effect exclusively at the level of performance and use of the mindreading ability. In consequence, language itselfas a part of cultural system - is not strictly a prerequisite for the development of the mindreading system. Such an interpretation is promoted by those who are in favor of the alternatives to the social-communicative approach. We can propose the following signature statements of this weak interpretation: (a) There exists an inborn mindreading mechanism that supplies all the propositional knowledge necessary for mindreading and is innately functioning already in early infancy; (b) the progression in mindreading that we track with ToM experiments is the outcome of improving interaction between this mindreading mechanism and other cognitive mechanisms that the system co-opts in more cognitively demanding tasks (Alan M. Leslie, 2010; Carruthers, 2013; Scholl \& Leslie, 1999).

Following this line of thought, Carruthers and Westra propose that the nature of cultural influence is such that children do have a universal, implicit understanding of other minds, but cultural experience enables them to voice it better and understand test questions in the proper way. In other words, the progression on the mindreading scale as well as cultural differences should be blamed on pragmatic misunderstandings of the implicatures of the test questions. For example, in a FB task, the child may interpret the question in a way that what the experimenter really is asking for is to help the character, not to exhibit knowledge. This is supported by the fact that young children are (i) chronically helpful, and that they have had little experience with (ii) knowledge-exhibiting questions and (iii) cognitive states 
as the topic of a conversation at that time of life (Westra \& Carruthers). This position, however, assumes - with no apparent ground for doing sothat cultural differences can significantly diversify a number of executive functions, while the whole conceptual system is insensitive to any change or cultural factors. It is said that it is not like the child does not mindread correctly, but rather that she misunderstands what is asked of her.

However, it can be tentatively pointed out here that not only crosscultural research but also mother-child relations research, as well as neuronal research demonstrate that simply adding a social environment to a preexisting mind draws a poor and incomplete picture. The idea that core concepts and competences are first established while cultural factors are only added to the child's cognitive repertoire clash not only with the ToM research, but also with research on modes of locomotion and number cognition. Such interpretation is therefore not tenable when we consider the above problems (Dehaene, Izard, Spelke, \& Pica, 2008; Gobel, Shaki, \& Fischer, 2011; Ngan Ng \& Rao, 2010).

\section{N O T E S}

1 This paper is supported by the National Science Center's grant (Poland) HARMONIA 6 (UMO-2014/14/M/HS1/00436 for years 2015-2018) for the research project entitled "The involvement of language, culture-specific factors and folk intuitions in mindreading activity and social cognition".

2 The theoretical framework at its most abstract iteration is called the systems theory. Other terms used are the relational-systems theory and the dynamic systems theory. Its application to development functions in the literature as the developmental systems approach. We will use them interchangeably, meaning the application of the framework to cognitive development, unless explicitly stating otherwise.

3 The approach is referred to in the literature as social, social-communicative, pragmatic, systemic, systems, dynamic systems and relational-systems. We will use the terms interchangeably in this general exposition.

\section{R E F E R E N C E S}

Alan M. Leslie. (2010). How to acquire a 'representational theory of mind'. In D. Sperber (Ed.), Vancouver studies in cognitive science: Vol. 10. Metarepresentations. A multidisciplinary perspective (pp. 197-223). Oxford: Oxford Univ. Press.

Alessandro Duranti. (2008). Further Reflections on Reading Other Minds. Anthropological Quarterly, 81(2), 483-494. doi:10.1353/anq.0.0002

Apperly, I. A., \& Butterfill, S. A. (2009). Do humans have two systems to track beliefs and belief-like states? Psychological review, 116(4), 953-970. doi: $10.1037 / \mathrm{a} 0016923$ 
Astington, J. W. (2006). The Developmental Inderdependence of Theory fo Mind and Language. In N. J. Enfield \& S. C. Levinson (Eds.), Wenner-Gren international symposium series. Roots of human sociality. Culture, cognition and interaction / edited by Nicholas J. Enfield and Stephen C. Levinson (pp. 179-206). Oxford: Berg.

Białecka-Pikul M., (2012) - Narodziny i rozwój refleksji nad myśleniem, Kraków: Wydawnictwo Uniwersytetu Jagiellońskiego.

Bogdan, R. J. (1997). Interpreting minds: The evolution of a practice / Radu J. Bogdan. A Bradford book. Cambridge, Mass., London: MIT Press.

Brown, J. R., Donelan-McCall, N., \& Dunn, J. (1996). Why Talk about Mental States?: The Significance of Children's Conversations with Friends, Siblings, and Mothers. Child Development, 67(3), 836. doi:10.2307/1131864

Callaghan, T., Rochat, P., Lillard, A., Claux, M. L., Odden, H., Itakura, S., Singh, S. (2005). Synchrony in the Onset of Mental-State Reasoning: Evidence From Five Cultures. Psychological Science, 16(5), 378-384. doi:10.1111 /j.0956-7976.2005.01544.x

Carpendale, J. I. M., \& Lewis, C. (2006). How children develop social understanding. Understanding children's worlds. Malden, Mass., Oxford: Blackwell.

Carruthers, P. (2013). Mindreading in Infancy. Mind \& Language, 28(2), 141-172. doi:10.1111/mila.12014

Dehaene, S., Izard, V., Spelke, E., \& Pica, P. (2008). Log or Linear?: Distinct Intuitions of the Number Scale in Western and Amazonian Indigene Cultures. Science, 320(5880), 1217-1220. doi:10.1126/science.1156540

Donald, M. (2006). A mind so rare: The evolution of human consciousness [Sound disc]. Princeton, N.J.: Recording for the Blind \& Dyslexic.

Dore, R. A., \& Lillard, A. S. (2014). Do Children Prefer Mentalistic Descriptions? The Journal of Genetic Psychology, 175(1), 1-15.

Furrow, D., Moore, C., Davidge, J., \& Chiasson, L. (1992). Mental terms in mothers' and children's speech: Similarities and relationships. Journal of Child Language, 19(03), 617. doi:10.1017/S0305000900011594

Gobel, S. M., Shaki, S., \& Fischer, M. H. (2011). The Cultural Number Line: A Review of Cultural and Linguistic Influences on the Development of Number Processing. Journal of Cross-Cultural Psychology, 42(4), 543-565. doi:10.1177/0022022111406251

Gopnik, A., Meltzoff, A. N., \& Kuhl, P. K. (1999). The scientist in the crib: What early learning tells us about the mind. Pymble, NSW, New York: HarperCollins e-books.

Gut, A. (2009). Language and Higher Order Thinking [Język a myślenie drugiego rzędu]. Filozofia Nauki, 17(3), 99-119. 
Gut, A. \& Wilczewski M. (2015). Is mindreading universal or cultural specific construct? In G. Airenti, B. G. Bara, G. Sandini, M. Cruciani (Eds.), Proceedings of the EuroAsianPacific Joint Conference on Cognitive Science (pp. 499505). Volume 1419 of CEUR Workshop Proceedings, CEUR-WS.org, 2015.

Hale, C. M., \& Tager-Flusberg, H. (2003). The influence of language on theory of mind: a training study. Developmental science, 6(3), 346-359. doi:10.1111/1467-7687.00289

Harris, P. (2005). Conversation, pretense and theory of mind. In J. W. Astington \& J. A. Baird (Eds.), Why language matters for theory of mind (pp. 70-83). New York, Oxford: Oxford University Press.

Hutto D.D., Herschbach M., Southgate V., (2011) - Social Cognition: Mindreading and Alternatives, "Review of Philosophical Psychology" 2, s. 275-395

Jenkins, J. M., \& Astington, J. W. (1996). Cognitive factors and family structure associated with theory of mind development in young children. Developmental Psychology, 32(1), 70-78. doi:10.1037/0012-1649.32.1.70

Joel Robbins, \& Alan Rumsey. (2008). Introduction: Cultural and Linguistic Anthropology and the Opacity of Other Minds. Anthropological Quarterly, 81(2), 407-420. doi:10.1353/anq.0.0005

Kallberg-Schroff M., \& Miller J.M. (2014). Culture and the development of Theory of Mind. In O. N. Saracho (Ed.), Contemporary Perspectives in Early Childhood Education. Contemporary perspectives on research in theory of mind in early childhood education (pp. 343-364). Charlotte, North Carolina: Information Age Publishing.

Kobayashi, C., Glover, G. H., \& Temple, E. (2006). Cultural and linguistic influence on neural bases of 'Theory of Mind': An fMRI study with Japanese bilinguals. Brain and Language, 98(2), 210-220. doi:10.1016/j.bandl.2006.04.013

Mayer, A., \& Trauble, B. E. (2012). Synchrony in the onset of mental state understanding across cultures?: A study among children in Samoa. International Journal of Behavioral Development, 37(1), 21-28. doi:10.1177/016502541245 4030

Meins, E., \& Fernyhough, C. (2010). Mind-mindedness coding manual: Version 2.0.

Milligan, K., Astington, J. W., \& La Dack. (2007). Language and theory of mind: meta-analysis of the relation between language ability and false-belief understanding. Child Development, 78(2), 622-646. doi:10.1111/j.1467-8624. 2007.01018.x

Nelson, K. (2007). Young minds in social worlds: Experience, meaning, and memory / Katherine Nelson. Cambridge, Mass., London: Harvard University Press.

Ngan Ng, S. S., \& Rao, N. (2010). Chinese Number Words, Culture, and Mathematics Learning. Review of Educational Research, 80(2), 180-206. doi:10.3102/0034654310364764

Ochs, E. (1988). Culture and language development: Language acquisition and language socialization in a Samoan village / Elinor Ochs. Studies in the social 
and cultural foundations of language: no. 6. Cambridge: Cambridge University Press.

Perner, J., Ruffman, T., \& Leekam, S. R. (1994). Theory of Mind Is Contagious: You Catch It from Your Sibs. Child Development, 65(4), 1228-1238. doi:10.1111/j.1467-8624.1994.tb00814.x

Pyers, J. E., \& Senghas, A. (2009). Language promotes false-belief understanding: evidence from learners of a new sign language. Psychological Science, 20(7), 805-812. doi:10.1111/j.1467-9280.2009.02377.x

Rosnay, M., \& Hughes, C. (2006). Conversation and theory of mind: Do children talk their way to socio-cognitive understanding? British Journal of Developmental Psychology, 24(1), 7-37. doi:10.1348/026151005X82901

Ruffman, T., Slade, L., \& Crowe, E. (2002). The relation between children's and mothers' mental state language and theory-of-mind understanding. Child Development, 73(3), 734-751.

Ruffman, T., Slade, L., Rowlandson, K., Rumsey, C., \& Garnham, A. (2003). How language relates to belief, desire, and emotion understanding. Cognitive Development, 18(2), 139-158. doi:10.1016/S0885-2014(03)00002-9

Scholl, B. J., \& Leslie, A. M. (1999). Modularity, Development and 'Theory of Mind'. Mind and Language, 14(1), 131-153. doi:10.1111/1468-0017.00106

Shahaeian, A., Peterson, C. C., Slaughter, V., \& Wellman, H. M. (2011). Culture and the sequence of steps in theory of mind development. Developmental Psychology, 47(5), 1239-1247. doi:10.1037/a0023899

Smith, B. H., Weintraub, E. R., Oyama, S., \& Lewontin, R. (Eds.). (2000). The Ontogeny of Information: Duke University Press.

Tafreshi, D., \& Racine, T. P. (2016). Children's interpretive theory of mind: The role of mothers' personal epistemologies and mother-child talk about interpretation. Cognitive Development, 39, 57-70. doi:10.1016/j.cogdev.2016.04. 003

Tager-Flusberg, H., \& Joseph, R. M. (2005). How Language Facilitates the Acquisition of False-Belief Understanding in Children with Autism. In J. W. Astington \& J. A. Baird (Eds.), Why language matters for theory of mind (pp. 298318). New York, Oxford: Oxford University Press. doi:10.1093/acprof:oso/97 80195159912.003.0014

Thelen, E., \& Smith, L. B. (2002). A dynamic systems approach to the development of cognition and action (5. print). A Bradford book. Cambridge, Mass.: MIT Press.

Villiers, J. G. de. (1995). Steps in the mastery of sentence complements. Paper presented at the Biennial Meeting of the Society for Research in Child Development. Indianapolis, IN.

Villiers, J. G. de. (2000). Language and Theory of mind: what are the developmental relationships?. In: S. Baron-Cohen, H. Tager-Flusberg and D. Cohen (Eds.) Understanding other Minds: Perspectives from Autism and Developmental Cognitive Neuroscience.Cambridge: Cambridge University Press, 83-123. 
Villiers, J. G. de, \& P. de Villiers (2000). Linguistic determinism and the understanding of false beliefs. In: P. Mitchell and K. Riggs (Eds.) Children's Reasoning and the Mind. Hove, UK: Psychology Press, 189-226.

Villiers, J. G. de, \& Pyers, J. E. (2002). Complements to cognition: A longitudinal study of the relationship between complex syntax and false-beliefunderstanding. Cognitive Development, 17(1), 1037-1060. doi:10.1016/S0885 -2014(02)00073-4

Villiers, J. G. de, \& Villiers, P. A. de. (2009). Complements Enable Representation of the Contents of False Beliefs: The Evolution of a Theory of Theory of Mind. In S. H. Foster-Cohen (Ed.), Palgrave advances in linguistics. Language acquisition (pp. 169-195). Houndmills, Basingstoke, Hampshire [England], New York: Palgrave Macmillan. doi:10.1057/9780230240780_8

Villiers, J. G. de, \& Villiers, P. A. de. (2014). The Role of Language in Theory of Mind Development. Topics in Language Disorders, 34(4), 313-328. doi:10.1097/TLD.0000000000000037

Villiers, P. A. de. (2005). The role of language in theory of mind development: What deaf children tell us. In J. W. Astington \& J. A. Baird (Eds.), Why language matters for theory of mind (pp. 266-297). New York, Oxford: Oxford University Press.

Wahi, S., \& Johri, R. (1994). Questioning a Universal Theory of Mind: Mental-Real Distinctions Made by Indian Children. The Journal of Genetic Psychology, 155(4), 503-510. doi:10.1080/00221325.1994.9914798

Watson, A. C., Nixon, C. L., Wilson, A., \& Capage, L. (1999). Social interaction skills and theory of mind in young children. Developmental Psychology, 35(2), 386-391. doi:10.1037/0012-1649.35.2.386

Wellman, H. M., Fang, F., Liu, D., Zhu, L., \& Liu, G. (2006). Scaling of Theoryof-Mind Understandings in Chinese Children. Psychological Science, 17(12), 1075-1081. doi:10.1111/j.1467-9280.2006.01830.x

Wellman, H. M., Fang, F., \& Peterson, C. C. (2011). Sequential Progressions in a Theory-of-Mind Scale: Longitudinal Perspectives. Child Development, 82(3), 780-792. doi:10.1111/j.1467-8624.2011.01583.x

Wellman, H. M., \& Liu, D. (2004). Scaling of theory-of-mind tasks. Child Development, 75(2), 523-541. doi:10.1111/j.1467-8624.2004.00691.x

Wellman, H. M., \& Peterson, C. C. (2013). Theory of mind, development, and deafness. In S. Baron-Cohen, H. Tager-Flusberg, \& M. Lombardo (Eds.), Understanding other minds. Perspectives from developmental social neuroscience / edited by Simon Baron-Cohen, Helen Tager-Flusberg and Michael Lombardo (3rd ed., pp. 51-71).

Westra, E. \& Carruthers, P. The theory-of-mind scale: A pragmatic approach. Retrieved from http://faculty.philosophy.umd.edu/pcarruthers/The\%20theoryof-mind $\% 20$ scale.pdf 